\title{
OPEN STANDARDS REQUIREMENTS ${ }^{1}$
}

\author{
Ken Krechmer, Fellow \\ International Center for Standards Research, University of Colorado \\ 757 Greer Road \\ Palo Alto, CA USA 94303-3024 \\ Tel: $+1-650-856-8836$ \\ Fax: +1-650-856-6591 \\ krechmer@csrstds.com \\ February 7, 2005
}

\footnotetext{
${ }^{1}$ An earlier revision of this paper was published in the Proceedings of the 38th Annual Hawaii International Conference on System Sciences, 2005. Both this paper and the version published in the HICSS Proceedings are major revisions of "The Principles of Open Standards", published in Standards Engineering, vol. 50, no. 6, November/December 1998.
} 


\title{
OPEN STANDARDS REQUIREMENTS
}

\author{
ABSTRACT \\ The personal computer revolution and the following internet wave have resulted in a large influx of \\ new technology stakeholders. These new stakeholders have a material interest in the technical \\ standards that proscribe their communications. These new stakeholders are making new demands on \\ the standardization processes, often with the rallying cry, "Open Standards." As is often the case, a \\ rallying cry means many different things to different people. This paper explores the different \\ requirements that the term open standards suggests. Perhaps when everyone agrees on what \\ requirements open standards serve, it will be possible to achieve them.
}

\section{INTRODUCTION}

Open standards, open source, open architecture -- all sound appealing, but what do they mean?

Some definitions are needed: Standards represent common agreements that enable communications, directly in the case of IT standards and indirectly in the case of all other standards. Open source is used to describe an open process of software development. Often open source development makes use of open standards for operating systems or software development tools, but the purpose of open source is to support continuous software improvement (Raymond, 2000) while the purpose of open standards is to support common agreements that enable communications open to all. Open 
architecture refers to a system whose internal and/or external interfaces are defined by open standards. The term Standards Setting Organization (SSO) refers to any and all organizations that set, or attempt to set, what the market perceives as standards. The term "recognized SSO" refers to any SSO recognized directly or indirectly by a government entity. Consortia is the term used for SSOs that are not recognized SSOs.

There are many requirements on a standard. The more basic requirements: consistent, logical, clear, etc., are easily agreed. This paper focuses on the requirements that emerge in information technology (IT) standards that are termed "open."

Standardization consists of more than the process of standards creation; standardization includes those who implement the standard (implementers) and those who use the implementations of the standard (users). As example, it is common for a user organization to say, "We have standardized on Microsoft Word," meaning that they have agreed to use Word in their organization. And Microsoft often refers to their implementation of Word as an open standard, meaning that they make their implementations of Word widely available to users (Gates, 1998). It seems clear that the term open standards, at least to some, includes the open implementation or open use of standards as well as the open creation of standards. 
Open Standards Requirements

This paper develops the argument that all technical communications standardization processes are in transition from being driven by standards creators (standardization participants who are motivated to develop new standards) to being driven by standards implementers (standardization participants who are motivated to produce new products that embody a standard). In addition, the users of standards (who usually do not participate in the initial IT standardization process) have a growing interest in seeing the concept of openness address their requirements.

As recognized SSOs developed in the late 19th century, they focused, often with government approval, on supporting the open creation of standards and not on the open implementation or open use of standards. This was quite reasonable as the standards stakeholders then were the creators of the standards. As example, the railroads were major creators, implementers and users of standards in this period. In the 19 th and early 20 th centuries, the significant standardization policy issue was the conversion from multiple company standards to single SSO standards (National Industrial Conference Board, 1929).

Through the middle of the 20th century, large integrated organizations (companies that bring together research and development, production and distribution of their products or services, e.g., IBM, AT\&T, Digital Equipment Corp, France Telecom, NTT) had engineers who functioned, often on a full time basis, as the integrated organization's standards creators. These standards creators 
Open Standards Requirements

supported specific, recognized SSOs necessary for the broad aims of the integrated organization (Cargill, 1989).

In the later 20th Century, as technology use and therefore the needed standards exploded, the number of implementers and users of standards increased dramatically and the stage was set for major changes in standardization processes and activity. By the middle of the 1980s, a new industrial movement emerged where larger integrated organizations began to devolve into segmented organizations (where the overall organization exerts the minimum unified management). Each segment organization focuses only on its market(s) and therefore only supports the SSOs that appeared necessary for their specific product development requirements (Updegrove, 1995). This new industrial movement marks the rise of the implementers' activity (independent product development group) in standardization. At the same time the overarching integrated organization's standardization organization was disbanded in many cases (examples include AT\&T, BellCore, and IBM).

Individual product development groups in segmented organizations have no history or allegiance to a specific SSO and choose to support any SSO that best fits their specific product development and marketing needs. Often such a fit is made by sponsoring a new SSO to address the standardization requirements of a specific developer's product implementation (Updegrove, 2004). As this paper explains, product implementers have very different interests than the standards creators they replaced. What a product implementer considers an open standard may be quite different from what 
a standards creator considers an open standard. Which may also be different from what a user might consider as an open standard.

\section{THE RECOGNIZED SSO VIEW OF OPEN STANDARDS}

Many SSOs' websites refer to the desirability of openness of standardization. There appears to be wide interest among recognized SSOs in open standards. Currently the recognized SSOs follow rules to ensure what they consider an open standards creation process by requiring open meetings, consensus and due process.

The Institute of Electrical and Electronic Engineers (IEEE) states, "For over a century, the IEEE-SA has offered an established standards development program that features balance, openness, due process, and consensus" (IEEE web site http://standards.ieee.org/sa/sa-view.html).

The European Telecommunications Standardization Institute (ETSI) web site explains: "The European model for telecom standardization allows for the creation of open standards" (ETSI web site http://www.etsi.org/\%40lis/background.htm).

The American National Standards Institute (ANSI) National Standards Strategy for the United States (2002) states, "The process to create these voluntary standards is guided by the Institute's cardinal 
Open Standards Requirements

principles of consensus, due process and openness ..." (ANSI web site

http://www.ansi.org/standards_activities/overview/overview.aspx?menuid=3).

However, the same recognized SSOs have few rules to ensure that procedures for the implementation or use of a standard are open. Even the idea that such procedures are even needed is quite new. Many recognized SSOs are comfortable addressing the standardization needs directly associated with the creation of standards and are still learning the needs of standards implementers and users.

\section{SOME DEFINITIONS OF OPEN STANDARDS}

West (2004) defines "'open' for a standard as meaning rights to the standard are made available to economic actors other than the sponsor." This definition offers a succinct economic view of open standards. But economic rights cannot not be maintained without supporting political rights such as balance, consensus and due process. In order for the economic rights associated with compatibility to be available, some technical process (change control) and technical functionality (open interfaces) are also required. In order for specific economic rights associated with Intellectual Property Rights (IPR) to be available, specific SSO procedures must be defined.

Perens (no date), the author of the Open Source definition, offers a software development perspective of open standards. He presents six requirements and related practices. The requirements 
Open Standards Requirements

proposed are: availability, maximize end-user choice, no royalty, no discrimination, extension or subset and predatory practices.

\section{THE TEN REQUIREMENTS THAT ENABLE OPEN STANDARDS}

This paper identifies and explains some of the different requirements that may be desired by creators, implementers and users of standards to understand what open standards means to all the stakeholders. The term "open standard" may be seen from these three perspectives:

- The recognized SSOs, as organizations representing the standards creators, considers a standard to be open if the creation of the standard follows the tenets of open meeting, consensus and due process.

- An implementer of an existing standard would call the standard open when it serves the market they wish, it is without cost to them, does not preclude further innovation (by them), does not obsolete their prior implementations, and does not favor a competitor.

- The user of an implementation of the standard would call a standard open when multiple implementations of the standard from different sources are available, when the implementation functions in all locations needed, when the implementation is supported over the user-planned service life, and when new implementations desired by the user are backward compatible to previously purchased implementations. 
Open Standards Requirements

This clarifies the very different views of the creators, implementers and users of standards on what is an open standard. Their combined, reasonable, but not simple, expectations translate into the ten requirements that enable open standards:

1. Open Meeting - all may participate in the standards development process.

2. Consensus - all interests are discussed and agreement found, no domination.

3. Due Process - balloting and an appeals process may be used to find resolution.

4. Open IPR - how holders of IPR related to the standard make available their IPR.

5. One World - same standard for the same capability, world-wide.

6. Open Change - all changes are presented and agreed in a forum supporting the five requirements above.

7. Open Documents - committee drafts and completed standards documents are easily available for implementation and use.

8. Open Interface - supports proprietary advantage (implementation); each interface is not hidden or controlled (implementation); each interface of the implementation supports migration (use).

9. Open Access - objective conformance mechanisms for implementation testing and user evaluation.

10. On-going Support - standards are supported until user interest ceases rather than when implementer interest declines. 
Open Standards Requirements

Standards are a multi-disciplinary field. The requirements of open standards should address each of the disciplines likely to describe them -- economics, law, engineering, social and political sciences. From the legal perspective, each of these ten requirements may be a legal right of a specific group. As West (2004) notes, each of these requirements has an economic cost and a benefit to specific stakeholders. From an engineering perspective, two of these requirements (6 and 8) directly impact communications equipment compatibility and design. From a social science perspective, the dynamics of different stakeholders may be examined in terms of each requirement. From a political science perspective, the first three requirements are basic to a fair political process.

Comparing these ten requirements to the six principles proposed by Perens (no date):

Availability is addressed by Open Documents.

Maximum end-user choice is addressed by Open Access.

No royalty is addressed under Open IPR.

No discrimination is addressed by Open Meeting, Consensus and Due Process.

Ability to create extension or subset is addressed by Open Interface.

Ability to prevent predatory practices is addressed by Open Change.

The six principles proposed by Perens (no date) map fully onto eight of the ten requirements of open standards proposed here. Perens does not directly address in the six principles the desires for or against One World or the end user requirement of on-going support. 


\section{THREE VIEWS OF STANDARDIZATION}

Each of the ten requirements of open standards relates to one or more of the stakeholders - creators, implementers and users. Each group of stakeholders are driven by specific economic needs:

- The creation of standards is driven by potential market development and control issues.

- The implementation of standards is driven by production and distribution cost efficiencies.

- The use of standards is driven by the potential efficiency improvement, due to the standard, on the user organization. In the case of standards used by political entities (e.g., countries), the use or avoidance of a standard may represent a political efficiency as well as an economic one.

While there is some overlap among these economic drivers, e.g., market development and distribution cost efficiency, each stakeholder has a distinct economic motivation. This makes it necessary to consider each stakeholder class separately. The relation of the ten requirements to the stakeholder classes is shown in Table 1, below. 
Open Standards Requirements

\begin{tabular}{|l|l|c|c|c|}
\cline { 3 - 5 } \multicolumn{2}{c|}{} & \multicolumn{3}{c|}{ Stakeholders } \\
\hline 1 & Open Meeting & Creator & Implementer & User \\
\hline 2 & Consensus & $\mathrm{x}$ & & \\
\hline 3 & Due Process & $\mathrm{x}$ & & \\
\hline 4 & One World & $\mathrm{x}$ & $\mathrm{x}$ & $\mathrm{x}$ \\
\hline 5 & Open IPR & $\mathrm{x}$ & $\mathrm{x}$ & $\mathrm{x}$ \\
\hline 6 & Open Change & $\mathrm{x}$ & $\mathrm{x}$ & $\mathrm{x}$ \\
\hline 7 & Open Documents & & $\mathrm{x}$ & $\mathrm{x}$ \\
\hline 8 & Open Interface & & $\mathrm{x}$ & $\mathrm{x}$ \\
\hline 9 & Open Access & & $\mathrm{x}$ \\
\hline 10 & On-going Support & & $\mathrm{x}$ \\
\hline
\end{tabular}

Table 1. Creators, implementers and users see openness differently.

Table 1 shows that the requirements of the major stakeholder groups are sometimes similar and sometimes divergent. Users have little interest in how a standardization process was conducted. The concept that open meetings, consensus and due process support the development of multiple sources of implementations of a completed standard is recognized but rarely supported by users. Users focus on being able to purchase compatible equipment from multiple sources. In the case of requirement 5 (Open IPR), even though all the stakeholders have an interest, their interests are quite different. Creators appear satisfied to support reasonable and non-discriminatory (RAND) IPR policies from the SSO. Implementers require a means to define their IPR cost, which RAND does 
not offer. This pushes implementers to form consortia. Users are often unwilling to pay high prices caused by multiple IPR claims.

As Table 1 identifies, the first three requirements are oriented to the stakeholders focused on standards creation. The fourth requirement, One World, is supported by ANSI but not required. The ANSI open standards concept requires the first three requirements for all ANSI accredited standards organizations (American National Standards Institute, 1998). The first four requirements are also at the heart of the World Trade Organization (WTO) Agreement on Technical Barriers to Trade, Code of Good Practice (WTO web site http://www.wto.org/english/tratop_e/tbt_e/tbtagr_e.htm\#Annex\%203). The fifth requirement, Open IPR, has been formally added to the US standards development process by ANSI and many SSOs.

Currently the greatest interest regarding open standards focuses on One World (requirement 4) and Open IPR (requirement 5). One World addresses standards as barriers to trade or as enablers of trade. Open IPR impacts the profitability of all communications equipment companies today. The additional five requirements (6 through 10) represent open standards requirements which are emerging, but are not yet supported by most SSOs. These additional requirements are oriented toward the implementation and use of standards.

\section{UNDERSTANDING THE TEN REQUIREMENTS OF OPEN STANDARDS}


Open Standards Requirements

In the following descriptions of the ten requirements, an attempt is made to quantify each specific requirement. Certainly the quantifications proposed are open to further consideration, but some quantification of each requirement is useful to identify how well different SSOs support the concept of open standards.

\section{Open Meeting}

"All stakeholders can participate" is a mantra of many recognized SSOs. But this mantra does not address all the barriers to open meetings. Recent social science research has identified 27 barriers to open meetings and grouped these into five categories: the stakeholders themselves, the rules of recognized standardization, the way the process is carried out, the role of the technical officers of the committee, and the culture of the committees (de Vries, Feilzer \& Verheul, 2004).

Currently openness of meetings is deemed to be met (e.g., under many SSO requirements) if all current stakeholders can participate in the standards creation process. But, as technology has become more complex, user participation in standards creation has declined (United States District Court, no date). When the largest group of stakeholders (users) no longer participates, such a definition of open meetings is no longer functional. 
Open Standards Requirements

The first barrier for stakeholders to participation in the standardization process is economic. Some recognized SSOs (e.g., ITU) and many consortia (e.g., W3C) have a pay-to-become-a-member policy. Paying to become a member is a significant economic barrier when a potential standardization participant is not sure they are even interested in attending a single meeting. Participation expenses, unless quite low, are part of real barriers to participation for students, many users and even start-up companies in the field. Currently only a few SSOs such as the Internet Engineering Task Force (IETF), the standardization organization for the Internet, and the IEEE, offer low cost per meeting participation.

Economic transparency may be a useful term to quantify open meetings. There are two broad indicators of the level of economic transparency possible in open meetings:

1. Any stakeholders can pay to become a member (current status of many SSOs).

2. Acceptable cost to join on a per meeting basis.

\section{Consensus}

Different SSOs define consensus differently. In general, consensus requires that no single stakeholder group constitutes a majority of the membership of an SSO. Consensus may be identified by vote of the standardization committee or may mean without active and informed opposition. Surprisingly, 
Open Standards Requirements

the IETF, which many find to be an example of a more open SSO, does not meet this criteria as the IETF Area Directors have a dictatorial level of control over the standardization decisions in their area (IETF Working Group Guidelines and Procedures, RFC 2418, September, 1998, http://www.ietf.org/rfc/rfc2418.txt).

Consensus is quantified in Table 3, below, only by the requirement (" 1 ") or lack of requirement ("0") in each SSO.

\section{Due Process}

Different SSOs describe due process differently. In general it requires that prompt consideration be given to the written views and objections of all participants. A readily available appeals mechanism for the impartial handling of procedural complaints regarding any action or inaction is part of the due process requirement.

Due process is quantified in Table 3, below, only by the requirement ("1") or lack of requirement ("0") in each SSO.

As explained above, the first three requirements, open meetings, consensus and due process, are considered fundamental by recognized SSOs to the openness of their standardization process. 
Open Standards Requirements

\section{Open World}

Open world is the principle of a single world-wide standard for a single purpose. This requirement is supported by the WTO to prevent technical barriers to trade. The International Federation of Standards Users (IFAN) also supports uniform international standards (IFAN strategies and policies for 2000-2005 http://www.ifan-online.org/). However, politically this can be a very contentious area. There are national standards for food processing that are based on religious beliefs (e.g., halal and kosher). There are standards for the environment, health, medical care, and social welfare that create an imbalance in cost between countries that implement them (richer) and countries that don't (poorer). To avoid these contentious issues, most recognized SSOs currently support, but do not require, coordination of their standards work with world-wide standards. This allows, but does not favor, divergent regional or national standards.

In the richer countries, the rise of consortia, the decline of publicly funded research, and aggressive commercialism make it more difficult to achieve a single standard for a single function world-wide. The five different incompatible wireless technologies of the $3 \mathrm{G}$ cellular standards (W-CDMA, cdma2000, UWC-136, TD-CDMA, FD-TDMA) are an example of these effects. Initially these five 3G versions will operate in different geographic areas but eventually users will demand world-wide compatibility. It appears likely that standardization organizations will continue to proliferate and 
Open Standards Requirements

create incompatible standards for similar capabilities. This may be viewed as an indication of the failings of recognized standardization (Cargill \& Bolin, 2004), or as an indication of the need to increase support of open interfaces (see requirement 8 , below).

Open world is quantified by identifying the geographic operating area of each SSO. The wider the jurisdiction of the SSO, the better the SSO will meet the open world requirement.

\section{Open IPR}

Most recognized SSOs and many consortia consider that open IPR means that holders of Intellectual Property Rights (IPR) must make their IPR for implementation available on Reasonable And NonDiscriminatory (RAND) terms. Five levels of quantification (“0” through "4") of open IPR currently are identified:

0. Commercial licensing may be the most prevalent way to use IPR legally. It is also the least open. In this case the holder of IPR and the potential implementer of the IPR agree privately on commercial terms and conditions for the implementer to use the holder's IPR.

Band (1995) describes four additional levels of increasing openness relating to IPR: 
Open Standards Requirements

1. Microsoft believes that interface specifications should be proprietary, but will permit openness by licensing the specifications to firms developing attaching (but not competing) products.

2. The Computer Systems Policy Project (CSPP) also believes that interface specifications can be proprietary, but will permit openness by licensing the specifications on RAND terms for the development of products on either side of the interface.

3. The American Committee for Interoperable Systems (ACIS) believes that software interface specifications are not protectable under copyright, and that therefore reverse engineering (including disassembly) to discern those specifications does not infringe the author's copyright.

4. Sun Microsystems believes that critical National Information Infrastructure (NII) software and hardware interface specifications should receive neither copyright nor patent protection. This fourth approach is discussed further under Open Change, below.

The above segmentation of IPR issues can be further refined. The approach 2 (the current manner of operation of most recognized SSOs) might be more acceptable to implementers if an IPR arbitration function exists when IPR is identified during the creation/modification of a standard (Shapiro, 2001). 
Open Standards Requirements

The approach 4 might be more acceptable to implementers if claims on basic interfaces were precluded but IPR on proprietary extensions was allowed. This could be technical practical using the concepts of open interfaces, below.

\section{Summary of the First Five Requirements}

The first three requirements of open standards have been addressed and in large measure resolved in most SSOs. The requirement for One World is certainly supported by the three world wide standardization organizations, ISO, IEC and ITU, but many nations cling to the view that giving up their national standardization prerogatives would be giving up an aspect of their nations' sovereignty. This creates another rationale for the rise of consortia.

Most recognized SSOs have not addressed the requirements of open standards beyond these first five requirements. This is a significant omission that can only be viewed negatively by those who are more interested in the implementation and use of standards.

The fifth requirement, Open IPR is the most divisive of the first five requirements as RAND is not sufficient to allow implementers to determine the impact of standards-based IPR on their costs. Semiconductors, where manufacturing costs drop rapidly with volume, exacerbate IPR problems for implementers. In the case of semiconductors, IPR costs may be based on fixed unit charges. Such 
Open Standards Requirements

changes can be the largest single cost component of a semiconductor. Semiconductor implementers must control their IPR costs. It seems only fair that any implementer have a right to determine the exact cost of IPR to them before they accept its inclusion in a new standard. This issue has led many implementers to consortia as consortia often require joint licensing of related IPR, which defines the cost of the IPR to the implementer. While commercial licensing is the least open process, it may not be more costly than the RAND approach. The advantages of commercial licensing versus the alternatives have led almost all implementers to utilize commercial licensing and to standardize with the consortia which require commercial licensing.

\section{Open Change}

Controlling changes is a powerful tool to control interfaces when system updates are distributed over the internet and stored in computer memory. Even with the most liberal of IPR policies identified (approach 4 in open IPR, above), Microsoft would still be able to control its Windows Application Programming Interfaces (APIs) by distributing updates (changes) to users that updated both sides of the API interface. Without a similar distribution at the same time, competing vendors' products on one side of the same API could be rendered incompatible by such a Microsoft update.

The only way that interfaces can remain "open" is when all changes are presented, evaluated and approved in a committee that supports the first five requirements. Considering today's environment 


\section{Open Standards Requirements}

of computers connected over the Internet, identifying and requiring open change is vital to the concept of open standards. Surprisingly, this is not widely understood. The original judicial order to break-up the Microsoft PC-OS and application software monopoly did not address this key issue(United States District Court for the District of Columbia Civil Action No. 98-1232 [TPJ]).

Consortia which do not support the first three requirements of open standards are the only SSOs that do not support open change.

\section{Open Documents}

Open documents is the requirement to see any documents from an SSO. The openness of a standardization meeting to outsiders is closely related to the availability of the documents from the meeting. All standardization documentation falls into two classes: work-in-progress documents (e.g., individual technical proposals, meeting reports, etc.), and completed standard documents (e.g., standards, test procedures, etc.). Different stakeholders need to access these different classes of documents. Standards creators do not require open documents as they are involved in the creation of all the documents. Standards implementers need access to standards work-in-progress documents, to understand specific technical decisions, as well as access to completed standards. Implementation testers (users and their surrogates) need access to completed standards. 


\section{Open Standards Requirements}

The Internet Society (ISOC) supports the IETF, which has pioneered new standards development and distribution procedures based on the internet itself. The IETF is a non-government-recognized standards making organization. While the IETF does not meet some criteria for consensus and due process, the IETF is perhaps the most transparent standardization organization. Using the internet, the IETF makes available on the web both its standards, termed RFCs, and the drafts of such standards at no charge. In fact, using the facilities of the internet, IETF committee discussion and individual technical proposals related to the development of standards can be monitored by anyone and response offered to the IETF during the standards creation process. This transparent development of IETF standards has been successful enough that some other SSOs are doing something similar. In July, 1998, ETSI announced that one technical committee TIPHON (Telecommunications and Internet Protocol Harmonization Over Networks) would make available at no charge all committee documents and standards drafts.

Ultimately, as technology use expands, everyone becomes stakeholders in technical standards. Using the internet, access to committee documents and discussion may be opened to almost all. In this way, informed choices may be made about bringing new work to a standards committee, and potential new standardization participants could evaluate their desires to attend meetings.

There are three levels of transparency in Open Documents: 


\section{Open Standards Requirements}

1. Work-in-progress documents are only available to committee members (standards creators).

Standards are for sale. (Current state of most formal SSOs.)

2. Work-in-progress documents are only available to committee members (standards creators).

Standards are available for little or no cost. (Current state of many consortia.)

3. Work-in-progress documents and standards are available for reasonable or no cost. (Current state of IETF.)

\section{Open Interface}

Open Interface is an emerging technical concept applicable to compatibility standards used between programmable systems. The open interface requirement supports compatibility to previous systems (backward compatibility) and to future systems (forward compatibility) that share the same interface. The idea that open standards should embody such a principle is new. But interest in open interfaces has been increasing due to the considerable success of open interfaces in facsimile (T.30), telephone modems (V.8 and V.32 auto baud procedures) and Digital Subscriber Line (DSL) transceivers (G.994.1).

One way of achieving open interfaces is to implement a new technique termed an "etiquette" (Krechmer, 2000). Etiquettes are a mechanism to negotiate protocols. While a protocol terminates an X.200 (OSI) layer, an etiquette which may negotiate multiple OSI layer protocols does not 
Open Standards Requirements

terminate (replace) any protocol layer function. An etiquette is used only for negotiating which protocol, options or features to employ. The purpose of etiquettes is connectivity and expandability. Proper etiquettes provide:

- Connectivity, negotiating between two devices in different spatial locations to determine compatible protocols.

- Means to allow both proprietary and public enhancements to the interface that do not impact backward or forward compatibility.

- Adaptability, so that a communications system can become compatible with a different communications system.

- Easier system troubleshooting by identifying specific incompatibilities.

As long as the etiquette is common between the equipment at both ends, or in the middle, it is possible to receive the code identifying each protocol supported by the equipment at a remote site. Checking this code against a data base of such codes on the web or in a manual, the user can determine what change is necessary in his system or the remote system to enable compatibility.

One of the earliest etiquettes is ITU Recommendation T.30 which is used in all Group 3 facsimile machines. Part of its function includes mechanisms to interoperate with previous Group 2 facsimile machines while allowing new features (public as well as proprietary) to be added to the system 


\section{Open Standards Requirements}

without the possibility of losing backward compatibility. In another case, the ITU standard V.8 is used to select among the V.34 and higher modem modulations. More recently, ITU G.994.1 was used to provide a similar function in Digital Subscriber Line equipment.

As an example of the usefulness of open interfaces, consider Microsoft APIs. Assume that a standard based upon the Microsoft Windows API is created. Then any vendor could create an operating system (OS) to work with Microsoft's applications or create applications to work with Microsoft's OS. If any vendor (including Microsoft) identified a new function such as short message service or video conferencing that was not supported across the basic API, that vendor could then offer the new function, as an identified proprietary feature across the API, to users that purchased the new vendor's OS and applications. Since an open interface supports proprietary extensions (Krechmer, 2000), each vendor controls the way the new function is accessed across the API, but does not change the basic compatibility of the API. In this manner a vendor is able to maintain control and add value, based on the desirability of the new function.

Krechmer (2000) identifies seven technical aspects of open interfaces. Currently open interfaces has only been applied at the standard committee level, not at the SSO level, so no detailed quantification is proposed in this paper.

\section{Open Access}


Open Standards Requirements

Open access describes the importance of accessibility by the users of standardized implementations (ANEC, the European consumer voice in standardization http://www.anec.org/). Accessibility divides into two classes: physical access (e.g., access by people with disabilities) and defined access (e.g., CE or UL mark indicating equipment is safe to use). Open access represents requirements on the standardization process as well as requirements on implementations that use the standard. Both may be monitored to assure conformance. To the implementer, some means is required to assure that their implementation of a standard works as they intended. To support this level of conformance, an interoperability event may be held (often termed a plug-fest). For the user, a simpler conformance indication is desirable. As example, in the European Union (EU), the CE marking is the manufacturer's indication that the product meets the essential safety requirements of all relevant EU Directives. This specific marking indicating safety conformance reduces the user's safety concerns. Two levels of open access are identified:

1. Open Access via plug-fests or over the internet testing (implementer).

2. Open Access via identified conformance (user). This may include the first level of access. 


\section{Open Standards Requirements}

\section{On-going Support}

On-going support of standards is of specific interest to standards users as it may increase the life of their capital investment in equipment with standard interfaces. The user's desire for implementer independent on-going support is noted by Perens (1999) as one of the desirable aspects of open source software. The support of an existing standard consists of four distinct phases after the standard is created (Table 2).

\begin{tabular}{|l|l|l|l|}
\hline Phase & Activity & Description & $\begin{array}{l}\text { Major Interest } \\
\text { Group }\end{array}$ \\
\hline 0. & Create standard & The major task of SSOs & Creators \\
\hline 1. & Fixes (changes) & $\begin{array}{l}\text { Rectify problems identified in initial } \\
\text { implementations }\end{array}$ & Implementers \\
\hline 2 & Maintenance (changes) & $\begin{array}{l}\text { Add new features and keep the standard } \\
\text { up to date with related standards work }\end{array}$ & Users \\
\hline 3. & $\begin{array}{l}\text { Availability } \\
\text { (no changes) }\end{array}$ & $\begin{array}{l}\text { maintenance } \\
\text { Removal of the published standard from } \\
\text { distribution }\end{array}$ & Users \\
\hline 4. & \begin{tabular}{l} 
Rescission \\
\hline
\end{tabular}
\end{tabular}

Table 2. The SSOs phases of support during a standard's lifetime.

This list may be used to quantify the on-going support that a specific SSO provides by identifying which steps of the on-going support process are widely announced by that SSO. This is a difficult requirement to quantify as different SSOs have different procedures for making this process public and many older SSOs do not make good use of the internet to distribute such information to users. 
It is difficult to interest users in the first phase of standards development (creation) shown in Table 2

(Naemura, 1995). Even the second phase, fixes, may be of more interest to the developers and implementers than the users. The next three phases, however, are where users have an interest in maintaining their investment. Possibly greater user involvement in the on-going support of standards would be practical by taking advantage of the internet to distribute standards and allow users to keep abreast of the work in standards meetings. Increasing the users' involvement with these aspects of the standardization process may also represent new economic opportunities for SSOs. Specific SSOs would be more valuable to users if the users recognized that the longevity of standards they had invested in was protected by that SSO.

\section{HOW OPEN ARE DIFFERENT SSOs?}

Table 3, below, offers the author's quantification, based on review of the SSO's documentation (as of 9/2004), of the specific requirements these SSOs support. By quantifying the 10 requirements of open standards, it is practical to examine any standardization processes to determine what requirements are supported, what requirements are not, and what economic shifts this causes. Then the political, social, economic, technical and practical implications of the process and the machinations of the stakeholders may be more rigorously analyzed and understood. 
Open Standards Requirements

\begin{tabular}{|l|l|l|l|l|l|l|l|}
\hline Reqmts. & $\begin{array}{l}\text { Consortia } \\
\text { (note 2) }\end{array}$ & $\begin{array}{l}\text { ITU } \\
\text { (note 1) }\end{array}$ & $\begin{array}{l}\text { IEEE } \\
\text { (note 1) }\end{array}$ & $\begin{array}{l}\text { ATIS T1 } \\
\text { (note 1) }\end{array}$ & $\begin{array}{l}\text { ETSI } \\
\text { (note 1) }\end{array}$ & W3C & IETF \\
\hline 1 OM & 1 & 1 & 2 & 1 & 1 & 1 & 2 \\
\hline 2 Con & 0 & 1 & 1 & 1 & 1 & 1 & 1 \\
\hline 3 DP & 0 & 1 & 1 & 1 & 1 & 0 & 0 \\
\hline 4 OW & 1 & 1 & 0 & 0 & 0 & 1 & 1 \\
\hline 5 OIPR & 0 & 2 & 2 & 2 & 2 & 4 & 3 (note 3) \\
\hline 6 OC & 0 & 1 & 1 & 1 & 1 & 1 & 1 \\
\hline 7 OD & 2 & 1 & 2 & 3 & 3 & 1 & 3 \\
\hline 8 OI & note 4 & note 4 & note 4 & note 4 & note 4 & note 4 & note 4 \\
\hline 9 OU & 2 (note 5) & 0 & 0 & 0 & 1 & 1 & 1 \\
\hline 10 OS & 1 & 2 & 2 & 2 & 4 & 4 & 4 \\
\hline Score & $\mathbf{7}$ & $\mathbf{1 0}$ & $\mathbf{1 1}$ & $\mathbf{1 1}$ & $\mathbf{1 4}$ & $\mathbf{1 4}$ & $\mathbf{1 6}$ \\
\hline
\end{tabular}

\section{Table 3. Rating "openness" of different SSOs.}

Note 1: The ITU, ETSI, IEEE, and ATIS are recognized SSOs.

Note 2: This hypothetical consortia is modeled on the description found at ConsortiumInfo.org (http://www.consortiuminfo.org/what).

Note 3: The IETF IPR policy desires a royalty free model, but is flexible.

Note 4: OI has only been applied to specific standards.

Note 5: Many consortia support plug-fests and conformance testing as part of their members desire to promote associated products.

\section{CONCLUSIONS}

Sometimes commercial organizations like to term their work "open standards" (Gates, 1998) when they meet few of the ten criteria identified. No SSOs discussed meet all of the ten requirements described and each SSO differs significantly in which requirements they do meet. So it should not be surprising that implementers and users consider all SSOs with a jaundiced eye. This attempt to rate 
Open Standards Requirements

how well SSOs support open standards suggests that this implementers' and users' view is a wise one.

While it is true that additional levels of each of the ten requirements may emerge, the ten basic requirements presented here are the broadest possible view of the meaning of open standards. Are fewer requirements sufficient? That question can only be answered when each stakeholder understands the consequences of what they may be giving up. The comprehension of the requirements that are supported by each SSO is usually buried in the fine print of the procedures of each SSO. Until each SSO clearly indicates which requirements of open standards they support and at what level, "open standards" will just be another marketing slogan.

The author would like to thank Joel West and Henk de Vries for their excellent comments and suggestions.

\section{REFERENCES}

American National Standards Institute (1998). Procedures for the Development and Coordination of American National Standards, April 1998. 


\section{Open Standards Requirements}

Band, J. (1995). “Competing Definitions of 'Openness' on the NII," Standards Policy for

Information Infrastructure, editors: Brian Kahin and Janet Abbate, The MIT Press, Cambridge, MA.

Cargill, C. (1989). Information Technology Standardization, page 114, Digital Press.

Cargill, C., and Bolin, S. (2004). "Standardization: a Failing Paradigm," paper presented at the Standards and Public Policy Conference, Federal Reserve Bank of Chicago, May 13-14, 2004.

de Vries, H., Feilzer, A. \& Verheul H. (2004). Removing Barriers for Participation in Formal Standardization. In: Françoise Bousquet, Yves Buntzly, Heide Coenen \& Kai Jakobs (Eds) EURAS Proceedings 2004. Aachener Beiträge zur Informatik, Band 36, Wissenschaftsverlag Mainz in Aachen, Aachen, pp. 171-176.

Gates, B. (1998). “Compete, Don't Delete,” The Economist, June 13, 1998.

Krechmer, K. (2000). “The Fundamental Nature of Standards: Technical Perspective,” IEEE Communications Magazine, Vol. 38 No. 6, p. 70. 
Open Standards Requirements

Naemura, K. (1995). "User involvement in the life cycles of information technology and telecommunications standards," Standards, Innovation and Competitiveness, editors: R. Hawkins, R. Mansell and J. Skea, Edward Elgar Publishing Limited.

National Industrial Conference Board Inc., (1929). Industrial Standardization, New York.

Perens, B. (1999). The Open Source Definition, C. DiBona, S. Ockman, \& M. Stone (eds.), OpenSources voices from the Open Source revolution, pages 171-189, Sebastopol, O'Reilly \& Associates.

Perens, B. (no date). Open Standards Principles and Practice http://perens.com/OpenStandards/Definition.html

Raymond, E. S. (2000). Homesteading the Noosphere, section 2, August 25, 2000. http://www.csaszar.org/interesting/the open_source_reader

Shapiro, C. (2001). "Setting Compatibility Standards Cooperation or Collusion," Expanding the Boundaries of Intellectual Property edited by R. C. Dreyfuss, D. L. Zimmerman and H. First, Oxford University Press. 


\section{Open Standards Requirements}

Updegrove, A. (1995). “Consortia and the Role of the Government in Standards Setting," Standards Policy for the Information Infrastructure, editors: Brian Kahin and Janet Abbate, The MIT Press, Cambridge MA.

Updegrove, A. (2004). "Best Practices and Standard Setting (How the "Pros" Do It)," The Standards Edge, Dynamic Tension, 2004, editor S. Bolin.

West, J. (2004). "What are Open Standards? Implications for Adoption, Competition and Policy," paper presented at the Standards and Public Policy Conference, Federal Reserve Bank of Chicago, May 13-14, 2004. 